\title{
THE RANK OF TRIFOCAL GRASSMANN TENSORS
}

\author{
MARINA BERTOLINI, GIAN MARIO BESANA, GILBERTO BINI, \\ AND CRISTINA TURRINI
}

\begin{abstract}
Grassmann tensors arise from classical problems of scene reconstruction in computer vision. Trifocal Grassmann tensors, related to three projections from a projective space of dimension $k$ onto view-spaces of varying dimensions are studied in this work. A canonical form for the combined projection matrices is obtained. When the centers of projections satisfy a natural generality assumption, such canonical form gives a closed formula for the rank of trifocal Grassmann tensors. The same approach is also applied to the case of two projections, confirming a previous result obtained with different methods in [6]. The rank of sequences of tensors converging to tensors associated with degenerate configurations of projection centers is also considered, giving concrete examples of a wide spectrum of phenomena that can happen.

Keywords. Tensor Rank, Border Rank, Projective reconstruction in Computer Vision, Multi-view Geometry.
\end{abstract}

\section{INTRODUCTION}

Tensors, as multidimensional arrays representing multilinear applications among vector spaces, have traditionally played a pivotal role in many areas, from physics to computer science, to electrical engineering. As algebraic geometry is increasingly witnessing intense activity in more applied directions, tensors have come to the fore of the discipline as useful tools on one hand, and as beautifully intricate objects of study on the other, with rich geometric interplay with other classical ideas. In particular, the calculation of any of the various established notions of rank of a tensor is an interesting and difficult problem. While many authors have recently studied these issues, a standard reference is [14] and a useful survey is 3 .

The authors have been interested for a while in a class of tensors that arise from classical problems of scene reconstruction in computer vision. In the classical case of reconstruction of a three-dimensional static scene from two, three, or four two-dimensional images, these tensors are known as the fundamental matrix, the trifocal tensor, and

Date: July 25, 2019. 
the quadrifocal tensor, respectively, and have been studied extensively, see for example [10], [1], [15], [2], [12]. In a more general setting, these tensors are called Grassmann tensors and were introduced by Hartley and Schaffalitzky, [11], as a way to encode information on corresponding subspaces in multiview geometry in higher dimensions. Three of the authors have studied critical loci for projective reconstruction from multiple views, [5], [8], and in this setting Grassmann tensors play a fundamental role, [7], [4].

The authors' long-term goal is to study properties such as rank, decomposition, degenerations, and identifiability of Grassmann tensors in higher dimensions, and, when feasible, the varieties parameterizing such tensors.

The first step was taken in [6], where three of the authors studied the case of two views in higher dimensions, introducing the concept of generalized fundamental matrices as 2-tensors. That first work contained an explicit geometric interpretation of the rational map associated to the generalized fundamental matrix, the computation of the rank of the generalized fundamental matrix with an explicit, closed formula, and the investigation of some properties of the variety of such objects.

The next natural step in the authors' program is the study of trifocal Grassmann tensors, i.e. Grassmann tensors arising from three projections from higher dimensional projective spaces onto view-spaces of varying dimensions. A natural genericity assumption, see Assumption 5.1, allows for suitable changes of coordinates in the view spaces and in the ambient space that give rise to a canonical form for the combined projection matrices. Utilizing such canonical form, the rank of trifocal Grassmann tensors is computed with a closed formula, see Theorem 5.1. When Assumption 5.1] is no longer satisfied, the situation becomes quite intricate. A general canonical form for the combined projection matrices can still be obtained, see Section 6. We conclude with a series of examples in which the rank is computed utilizing the canonical form. These examples illustrate the wide spectrum of possible phenomena that can happen with the specialization of the three centers of projection. In particular, we provide examples of sequences of Grassmann tensors of given rank $r$, converging to limit tensors whose rank can be either strictly larger than $r$, Example 6.2. and Example 6.3-a, or strictly smaller than $r$, Example 6.3-b. The first two of these cases are geometric examples of tensors with border rank strictly smaller than their rank.

\section{Background Material}


2.1. Preliminaries on tensors. Notation and definitions of tensors and their ranks (rank and border-rank) used in this work are relatively standard in the literature. They are all contained in [14] and briefly summarized below.

Given vector spaces $V_{i}, i=1, \ldots t$, the rank of a tensor $T \in V_{1} \otimes V_{2} \otimes$ $\ldots \otimes V_{t}$, denoted by $R(T)$, is the minimum number of decomposable tensors needed to write $T$ as a sum. Recall that $R(T)$ is invariant under changes of bases in the vector spaces $V_{i}$ (see for example [14], Section 2.4 ).

Furthermore, a tensor $T$ has border rank $r$ if it is a limit of tensors of rank $r$ but is not a limit of tensors of rank $s$ for any $s<r$. Let $\underline{R}(\mathcal{T})$ denote the border rank of $T$. Note that $\underline{R}(\mathcal{T}) \leq R(T)$.

As in Section 4 we will focus on tri-linear tensors, we recall here that given a tensor $T \in V_{1} \otimes V_{2} \otimes V_{3}$, where $\operatorname{dim} V_{i}=a_{i}$, its rank $R(T)$ can also be realized as the minimal number $p$ of rank $1 a_{1} \times a_{2^{-}}$ matrices $S_{1}, \ldots, S_{p}$ such that each slice $T_{i, j, \hat{k}}$, for a fixed $\hat{k}$, is a linear combination of such $S_{1}, \ldots, S_{p}$ (see for example [9], Theorem 2.1.2.).

2.2. Multiview Geometry. For the convenience of the reader, in this Section we recall standard facts and notation for cameras, centers of projection, and multiple views in the context of projective reconstruction in computer vision. A scene is a set of $N$ points $\left\{\mathbf{X}_{i}\right\} \in \mathbb{P}^{k}, i=$ $1, \ldots, N$. A camera $P$ is a projection from $\mathbb{P}^{k}$ onto $\mathbb{P}^{h},(h<k)$, from a linear center $C_{P}$. The target space $\mathbb{P}^{h}$, is called view. Once homogeneous coordinates have been chosen in $\mathbb{P}^{k}$ and $\mathbb{P}^{h}, P$ can be identified with a $(h+1) \times(k+1)$ - matrix of maximal rank, defined up to a constant, for which we use the same symbol $P$. With this notation, $C_{P}$ is the right annihilator of $P$, hence a $(k-h-1)$-space. Accordingly, if $\mathbf{X}$ is a point in $\mathbb{P}^{k}$, we denote its image in the projection equivalently as $P(\mathbf{X})$ or $P \cdot \mathbf{X}$.

The rows of $P$ represent linear subspaces of $\mathbb{P}^{k}=\mathbb{P}\left(\mathbb{C}^{k+1}\right)$ defining the center of projection $C_{P}$ and can be identified with points of the dual space $\breve{\mathbb{P}}^{k}=\mathbb{P}\left(\check{\mathbb{C}}^{k+1}\right)$, within which they span a linear space of dimension $h, \Lambda_{P}=\mathbb{P}\left(L_{P}\right)$, where $L_{P}$ is a complex vector space of dimension $h+1$.

The right action of $G L(k+1)$ on $P$ corresponds to a change of coordinates in $\mathbb{P}^{k}$, while the left action of $G L(h+1)$ can be thought of either as a change of coordinates in $L_{P}$ or in the view.

In the context of multiple view geometry, one considers a set of multiple images of the same scene, obtained from a set of cameras $P_{j}: \mathbb{P}^{k} \backslash C_{P_{j}} \rightarrow \mathbb{P}^{h_{j}}$.

Two different images $P_{l}(\mathbf{X})$ and $P_{m}(\mathbf{X})$ of the same point $\mathbf{X}$ are corresponding points and, more generally, $r$ linear subspaces $\mathcal{S}_{j} \subset \mathbb{P}^{h_{j}}$, 
$j=1, \ldots, r$ are said to be corresponding if there exists at least one point $\mathbf{X} \in \mathbb{P}^{k}$ such that $P_{j}(\mathbf{X}) \in \mathcal{S}_{j}$ for $j=1, \ldots, r$.

2.3. Grassmann Tensors. In the context of multiview geometry, Hartley and Schaffalitzky, [11], introduced Grassmann tensors, which encode the relations between sets of corresponding subspaces in the various views. We recall here the basic elements of their construction.

Consider a set of projections $P_{j}: \mathbb{P}^{k} \backslash C_{P_{j}} \rightarrow \mathbb{P}^{h_{j}}, j=1, \ldots, r, h_{j} \geq 2$ and a profile, i.e. a partition $\left(\alpha_{1}, \alpha_{2}, \ldots, \alpha_{r}\right)$ of $k+1$, where $1 \leq \alpha_{j} \leq h_{j}$ for all $j$, and $\sum \alpha_{j}=k+1$.

Let $\left\{\mathcal{S}_{j}\right\}, j=1, \ldots, r$, where $\mathcal{S}_{j} \subset \mathbb{P}^{h_{j}}$, be a set of general $s_{j}$-spaces, with $s_{j}=h_{j}-\alpha_{j}$, and let $S_{j}$ be the maximal rank $\left(h_{j}+1\right) \times\left(s_{j}+\right.$ 1)-matrix whose columns are a basis for $\mathcal{S}_{j}$. By definition, if all the $\mathcal{S}_{j}$ are corresponding subspaces there exist a point $\mathbf{X} \in \mathbb{P}^{k}$ such that $P_{j}(\mathbf{X}) \in \mathcal{S}_{j}$ for $j=1, \ldots, r$. In other words there exist $r$ vectors $\mathbf{v}_{\mathbf{j}} \in$ $\mathbb{C}^{s_{j}+1} j=1, \ldots, r$, such that:

$$
\left[\begin{array}{ccccc}
P_{1} & S_{1} & 0 & \ldots & 0 \\
P_{2} & 0 & S_{2} & \ldots & 0 \\
\vdots & \vdots & \vdots & \vdots & \vdots \\
P_{r} & 0 & \ldots & 0 & S_{r}
\end{array}\right] \cdot\left[\begin{array}{c}
\mathbf{X} \\
\mathbf{v}_{\mathbf{1}} \\
\mathbf{v}_{\mathbf{2}} \\
\vdots \\
\mathbf{v}_{\mathbf{r}}
\end{array}\right]=\left[\begin{array}{c}
0 \\
0 \\
\vdots \\
0
\end{array}\right]
$$

The existence of a non trivial solution $\left\{\mathbf{X}, \mathbf{v}_{\mathbf{1}}, \ldots, \mathbf{v}_{\mathbf{r}}\right\}$ for system (1) implies that the system matrix has zero determinant. This determinant can be thought of as an $r$-linear form, i.e. a tensor, in the Plücker coordinates of the spaces $\mathcal{S}_{j}$. This tensor is called the Grassmann tensor $\mathcal{T}$, and $\mathcal{T} \in V_{1} \otimes V_{2} \otimes \ldots \otimes V_{r}$ where $V_{i}$ is the $\left(\begin{array}{c}h_{i}+1 \\ h_{i}-\alpha_{i}+1\end{array}\right)$ vector space such that $G\left(s_{i}, h_{i}\right) \subset \mathbb{P}\left(V_{i}\right)$. More explicitly, the entries of the Grassmann tensor are some of the Plücker coordinates of the matrix:

$$
\left[P_{1}^{T}\left|P_{2}^{T}\right| \ldots \mid P_{r}^{T}\right]
$$

indeed they are, up to sign, the maximal minors of the matrix (2) obtained selecting $\alpha_{i}$ columns from $P_{i}^{T}$, for $i=1, \ldots, r$.

It is useful to observe the effect on a Grassmann tensor and its rank of the actions of $G L(k+1)$ on the ambient space and of $G L\left(h_{i}+1\right)$ on the views. A change of coordinates in the ambient space, realized by a right action of $G L(k+1)$ on (2) does not alter the tensor, as all entries are multiplied by the same non-zero constant. On the other hand, any change of coordinates in a view through left action of $G L\left(h_{i}+1\right)$ on the corresponding $P_{i}^{T}$ does alter the entries of the tensor, but preserves its rank. Indeed, the change of coordinates in one of the views induces 
a linear invertible transformation on $V_{i}$, leaving the rank unchanged, as noted in Section 2.1.

In the following Sections we deal with the cases of two and three views, in which the Grassmann tensor turns out to be respectively a matrix and a three dimensional tensor.

\section{Generalized Fundamental matrix}

We consider here the case of two views which gives rise to the notion of generalized fundamental matrix, introduced and studied in [6]. Let us consider two maximal rank projections $A=\left[a_{i, j}\right]$ and $B=\left[b_{i, j}\right]$ from $\mathbb{P}^{k}$ to $\mathbb{P}^{h_{1}}$ and to $\mathbb{P}^{h_{2}}$, respectively, where $h_{1}+h_{2} \geq k+1$, and where $A$ and $B$ are such that their projection centers $C_{A}$ and $C_{B}$ are in general position so that they do not intersect. This condition is equivalent to the fact that the linear span $\left\langle L_{A}, L_{B}\right\rangle$ is the whole $\check{\mathbb{C}}^{k+1}$. The images of the two centers of projection $E_{B}^{A}=A\left(C_{B}\right)$ and $E_{A}^{B}=B\left(C_{A}\right)$ are subspaces of dimension $k-h_{i}-1, i=1,2$, respectively, of the view spaces, usually called epipoles.

Following [11], we choose a profile $\left(\alpha_{1}, \alpha_{2}\right)$, with $\alpha_{1}+\alpha_{2}=k+1$, in order to obtain the constraints necessary to determine the corresponding tensor, which, in this case, is a matrix called generalized fundamental matrix. In the following we make explicit how to place the minors of (2) as entries of the generalized fundamental matrix.

In this case, (2) becomes

$$
\left[A^{T} \mid B^{T}\right]
$$

and the generalized fundamental matrix $\mathfrak{F}$ is the $\left(\begin{array}{c}h_{1}+1 \\ h_{1}-\alpha_{1}+1\end{array}\right) \times\left(\begin{array}{c}h_{2}+1 \\ h_{2}-\alpha_{2}+1\end{array}\right)$ matrix, whose entries are some of the Plücker coordinates of the $k$-space $\Lambda_{A B} \subset \mathbb{P}^{h_{1}+h_{2}+1}$, spanned by the columns of the above matrix.

Let $I=\left(i_{1}, \ldots, i_{s_{1}+1}\right), J=\left(j_{1}, \ldots, j_{s_{2}+1}\right), \hat{J}=\left(h_{1}+1+j_{1}, \ldots, h_{1}+\right.$ $\left.1+j_{s_{2}+1}\right)$ with $1 \leq i_{1}<\cdots<i_{s_{1}+1} \leq h_{1}+1$ and $1 \leq j_{1}<\cdots<$ $j_{s_{2}+1} \leq h_{2}+1$. Denote by $I^{\prime}, \hat{J}^{\prime}$ the (ordered) sets of complementary indices $I^{\prime}=\left\{r \in\left\{1, \ldots, h_{1}+1\right\}\right.$ such that $\left.r \notin I\right\}$ and $\hat{J}^{\prime}=\{s \in$ $\left\{h_{1}+2, \ldots, h_{1}+h_{2}+2\right\}$ such that $\left.s \notin \hat{J}\right\}$. Moreover denote by $A_{I}$ and $B_{J}$ the matrices obtained from $A^{T}$ and $B^{T}$ by deleting columns $i_{1}, \ldots, i_{s_{1}+1}$ and $j_{1}, \ldots, j_{s_{2}+1}$, respectively.

Then the entries of $\mathfrak{F}$ are: $F_{I, J}=\epsilon(I, J) \operatorname{det}\left[\begin{array}{ll}A_{I} & B_{J}\end{array}\right]$ where $\epsilon(I, J)$ is +1 or -1 according to the parity of the permutation $\left(I, \hat{J}, I^{\prime}, \hat{J}^{\prime}\right)$, with lexicographical order of the multi-indices $\{I\}$ for the rows and $\{\hat{J}\}$ for the columns. 
In other words, one has $F_{I, J}=q_{I, \hat{J}}\left(\Lambda_{A B}\right)$, where $q_{K}(\Lambda)$ denotes the dual-Plücker coordinates (see, for example, [13], Vol.I, book II, pg. 292) of the space $\Lambda$, with respect to the multi-index $K$.

In [6] the authors proved the following result:

Theorem 3.1. The generalized fundamental matrix $\mathfrak{F}$ for two projections of maximal rank and whose centers do not intersect each other, with profile $\left(\alpha_{1}, \alpha_{2}\right)$, has rank:

$$
\operatorname{rk}(\mathfrak{F})=\left(\begin{array}{c}
\left(h_{1}-\alpha_{1}+1\right)+\left(h_{2}-\alpha_{2}+1\right) \\
h_{1}-\alpha_{1}+1
\end{array}\right) .
$$

The proof given in [6] is obtained associating to the matrix $\mathfrak{F}$ a rational map $\Phi: G\left(s_{1}, h_{1}\right) \rightarrow G\left(k-\alpha_{1}, h_{2}\right)$ whose image is the Schubert variety $\Omega\left(E_{A}^{B}\right)$ of the $k-\alpha_{1}$ spaces containing $E_{A}^{B}$, and showing that $\operatorname{rk}(\mathfrak{F})=\operatorname{dim}\left(<\Omega\left(E_{A}^{B}\right)>\right)+1$, where $<\Omega\left(E_{A}^{B}\right)>$ is the projective space spanned by $\Omega\left(E_{A}^{B}\right)$.

In view of desired generalizations, here we give a straightforward proof of Theorem 3.1 based on a suitable choice of coordinates in the projective spaces involved.

Let $L_{A}$ and $L_{B}$ be the two vector spaces of dimension $h_{1}+1$ and $h_{2}+1$,respectively, spanned by the columns of $A^{T}$ and $B^{T}$ and let $\Lambda_{A}=\mathbb{P}\left(L_{A}\right)$ and $\Lambda_{B}=\mathbb{P}\left(L_{B}\right)$. We denote with $i$ the dimension of $I_{A, B}:=L_{A} \cap L_{B}$ which, from Grassmann's formula, turns out to be $i=h_{1}+h_{2}-k+1$. Notice that our assumptions on the profile $(k+1=$ $\left.\alpha_{1}+\alpha_{2}\right)$ imply that $i>0$.

One can then choose bases

$$
\begin{aligned}
& \left\{v_{1}, \ldots, v_{i}, w_{i+1}, \ldots, w_{h_{1}+1}\right\} \text { for } L_{A} \\
& \left\{v_{1}, \ldots, v_{i}, w_{i+1}^{\prime}, \ldots, w_{h_{2}+1}^{\prime}\right\} \text { for } L_{B}
\end{aligned}
$$

such that $\left\{v_{1}, \ldots, v_{i}\right\}$ is a basis for $I_{A, B}$.

Through the left action of $G L\left(h_{1}+1\right)$ and $G L\left(h_{2}+1\right)$ on $A$ and $B$ respectively, one can then assume that the columns of $A^{T}$ and $B^{T}$ are , respectively,

$$
\left[v_{1}, \ldots, v_{i}, w_{i+1}, \ldots, w_{h_{1}+1}\right]
$$

and

$$
\left[v_{1}, \ldots, v_{i}, w_{i+1}^{\prime}, \ldots, w_{h_{2}+1}^{\prime}\right]
$$

With this assumption, $\left\{v_{1}, \ldots, v_{i}, w_{i+1}, \ldots, w_{h_{1}+1}, w_{i+1}^{\prime}, \ldots, w_{h_{2}+1}^{\prime}\right\}$ is a basis of $\check{\mathbb{C}}^{k+1}$, and, with the right action of $G L(k+1)$, we can reduce it to the canonical one $\left\{e_{1}, \ldots, e_{k+1}\right\}$. With this choice, the 
matrix (3) becomes the block matrix

$$
\Phi_{h_{1}, h_{2}}^{k}:=\left[\begin{array}{cc|cc}
I_{i} & \mathbf{0} & I_{i} & \mathbf{0} \\
\mathbf{0} & I_{h_{1}+1-i} & \mathbf{0} & \mathbf{0} \\
\mathbf{0} & \mathbf{0} & \mathbf{0} & I_{h_{2}+1-i}
\end{array}\right]
$$

where $I_{s}$ denotes the $s \times s$ identity matrix and $\mathbf{0}$ are zero matrices.

The columns of $\Phi_{h_{1}, h_{2}}^{k}$ are denoted by:

$$
\left[\begin{array}{lllllllllll}
\underline{a}_{1} & \cdots & \underline{a}_{i} & \underline{b}_{i+1} & \cdots & \underline{b}_{h_{1}+1} \mid \underline{c}_{h_{1}+2} & \cdots & \underline{c}_{h_{1}+1+i} & \underline{d}_{h_{1}+2+i} & \cdots & \underline{d}_{h_{1}+h_{2}+2}
\end{array}\right]
$$

With this choice of basis, the entries of the fundamental matrix are the maximal minors of $\Phi_{h_{1}, h_{2}}^{k}$ obtained with $\alpha_{1}$ columns chosen among the $\underline{a}_{j}$ and $\underline{b}_{j}$ and $\alpha_{2}$ columns chosen among the $\underline{c}_{j}$ and $\underline{d}_{j}$. The only non vanishing entries of the fundamental matrix are hence obtained taking all the columns $\underline{b}_{j}$ and $\underline{d}_{j}$ and choosing $\alpha_{1}-\left(h_{1}+1-i\right)$ columns among the $\underline{a}_{j}$ and the complementary $\alpha_{2}-\left(h_{2}+1-i\right)$ among the $\underline{c}_{j}$. It follows that the non vanishing entries are as many as the possible choices of $\alpha_{1}-\left(h_{1}+1-i\right)$ columns among the first $i$ columns of $\Phi_{h_{1}, h_{2}}^{k}$. In other words the non zero entries of the fundamental matrix are:

$$
\left(\begin{array}{c}
i \\
h_{2}-\alpha_{2}+1
\end{array}\right)=\left(\begin{array}{c}
\left(h_{1}-\alpha_{1}+1\right)+\left(h_{2}-\alpha_{2}+1\right) \\
h_{1}-\alpha_{1}+1
\end{array}\right) .
$$

This number is precisely the rank of the fundamental matrix since non vanishing entries appear in different rows and columns of the fundamental matrix.

To clarify the above procedure we consider the following example.

Example 3.1. Consider two projections from $\mathbb{P}^{4}$ to $\mathbb{P}^{3}$ with profile $(3,2)$. In this case the matrix (3) has dimension $5 \times 8$. The subspace $\Lambda_{A B}$ is in $G(4,7) \subset \mathbb{P}^{\left(\begin{array}{l}8 \\ 5\end{array}\right)-1}$, and the fundamental matrix $\mathfrak{F}$ turns out to be:

$$
\mathfrak{F}=\left[\begin{array}{llllll}
q_{1,5,6} & q_{1,5,7} & q_{1,5,8} & q_{1,6,7} & q_{1,6,8} & q_{1,7,8} \\
q_{2,5,6} & q_{2,5,7} & q_{2,5,8} & q_{2,6,7} & q_{2,6,8} & q_{2,7,8} \\
q_{3,5,6} & q_{3,5,7} & q_{3,5,8} & q_{3,6,7} & q_{3,6,8} & q_{3,7,8} \\
q_{4,5,6} & q_{4,5,7} & q_{4,5,8} & q_{4,6,7} & q_{4,6,8} & q_{4,7,8}
\end{array}\right]
$$

and the matrix $\Phi_{3,3}^{4}$ is:

$$
\Phi_{3,3}^{4}=\left[\begin{array}{llll|llll}
1 & 0 & 0 & 0 & 1 & 0 & 0 & 0 \\
0 & 1 & 0 & 0 & 0 & 1 & 0 & 0 \\
0 & 0 & 1 & 0 & 0 & 0 & 1 & 0 \\
0 & 0 & 0 & 1 & 0 & 0 & 0 & 0 \\
0 & 0 & 0 & 0 & 0 & 0 & 0 & 1
\end{array}\right]
$$


so that the generalized fundamental matrix, in canonical form, is the following, from which it is evident that $\operatorname{rk}(\mathfrak{F})=3$ :

$$
\mathfrak{F}_{\mathfrak{C}}=\left[\begin{array}{cccccc}
0 & 0 & 0 & \pm 1 & 0 & 0 \\
0 & \pm 1 & 0 & 0 & 0 & 0 \\
\pm 1 & 0 & 0 & 0 & 0 & 0 \\
0 & 0 & 0 & 0 & 0 & 0
\end{array}\right]
$$

\section{Trifocal Grassmann tensors}

Let us now consider three projections $P_{1}, P_{2}$, and $P_{3}$, from $\mathbb{P}^{k}$ to $\mathbb{P}^{h_{1}}$, $\mathbb{P}^{h_{2}}$ and to $\mathbb{P}^{h_{3}}$, respectively, where $h_{1}+h_{2}+h_{3} \geq k+1$, and where $P_{1}, P_{2}$, and $P_{3}$, are maximal rank matrices.

Grassmann's formula shows that for generic choices of $P_{1}, P_{2}$, and $P_{3}$, their projection centers $C_{1}, C_{2}$, and $C_{3}$ are mutually disjoint under the assumptions: $k-h_{i}+h_{j}-1 \leq 0$, for $1 \leq i, j \leq 3, i \neq j$.

As in the case of the generalized fundamental matrix, let $\left(\alpha_{1}, \alpha_{2}, \alpha_{3}\right)$, be a profile with $\alpha_{1}+\alpha_{2}+\alpha_{3}=k+1$, in order to obtain the necessary constraints to determine the corresponding tensor. The tensor thus obtained is called the trifocal Grassman tensor and it is a generalization of the classical trifocal tensor for three views in $\mathbb{P}^{3}$. Its entries can be explicitly computed from (11), as shown below.

In this case, (2) becomes

$$
\left[P_{1}^{T}\left|P_{2}^{T}\right| P_{3}^{T}\right]
$$

and the entries of the trifocal tensor $\mathcal{T}$ are, up to sign, some of the maximal minors of the matrix (4) obtained by choosing $\alpha_{1}$ columns in $P_{1}^{T}, \alpha_{2}$ in $P_{2}^{T}$ and $\alpha_{3}$ in $P_{3}^{T}$.

More explicitly, let $I=\left(i_{1}, \ldots, i_{s_{1}+1}\right), J=\left(j_{1}, \ldots, j_{s_{2}+1}\right), K=$ $\left(k_{1}, \ldots, k_{s_{3}+1}\right), \hat{J}=\left(h_{1}+1+j_{1}, \ldots, h_{1}+1+j_{s_{2}+1}\right)$ and $\hat{K}=\left(h_{1}+\right.$ $\left.h_{2}+2+k_{1}, \ldots, h_{1}+h_{2}+2+k_{s_{3}+1}\right)$ with $1 \leq i_{1}<\cdots<i_{s_{1}+1} \leq h_{1}+1$, $1 \leq j_{1}<\cdots<j_{s_{2}+1} \leq h_{2}+1$ and $1 \leq k_{1}<\cdots<k_{s_{3}+1} \leq h_{3}+1$.

Denote by $I^{\prime}, \hat{J}^{\prime}, \hat{K}^{\prime}$ the (ordered) sets of complementary indices $I^{\prime}=$ $\left\{r \in\left\{1, \ldots, h_{1}+1\right\}\right.$ such that $\left.r \notin I\right\}$ and $\hat{J}^{\prime}=\left\{s \in\left\{h_{1}+2, \ldots, h_{1}+\right.\right.$ $\left.h_{2}+2\right\}$ such that $\left.s \notin \hat{J}\right\}$ and $\hat{K}^{\prime}=\left\{t \in\left\{h_{1}+h_{2}+3, \ldots, h_{1}+h_{2}+\right.\right.$ $\left.h_{3}+3\right\}$ such that $\left.t \notin \hat{K}\right\}$. Moreover denote by $P_{1 I}, P_{2 J}$ and $P_{3 K}$ respectively, the matrices obtained from $P_{1}^{T}, P_{2}^{T}$ and $P_{3}^{T}$ deleting columns $i_{1}, \ldots, i_{s_{1}+1}, j_{1}, \ldots, j_{s_{2}+1}$ and $k_{1}, \ldots, k_{s_{3}+1}$, respectively. Let $\epsilon\left(i_{1}, \ldots, i_{n}\right)$ be +1 or -1 according to the parity of the permutation 
$\left(i_{1}, \ldots, i_{n}\right)$. The entries of $\mathcal{T}$ are given by:

$$
\mathcal{T}_{I, J, K}=\epsilon\left(I, \hat{J}, \hat{K}, I^{\prime}, \hat{J}^{\prime}, \hat{K}^{\prime}\right) \operatorname{det}\left[\begin{array}{l}
P_{1 I} \\
P_{2 J} \\
P_{3 K}
\end{array}\right]
$$

Denote by $V_{i}$ the vector space such that $G\left(s_{i}, h_{i}\right) \subseteq \mathbb{P}^{\left(\begin{array}{l}h_{i}+1 \\ s_{i}+1\end{array}\right)-1}=$ $\mathbb{P}\left(V_{i}\right)$. The trifocal Grassmann tensor for three projections $P_{1}, P_{2}, P_{3}$ from $\mathbb{P}^{k}$ to $\mathbb{P}^{h_{1}}, \mathbb{P}^{h_{2}}$ and $\mathbb{P}^{h_{3}}$, with profile $\left(\alpha_{1}, \alpha_{2}, \alpha_{3}\right)$, is, up to a multiplicative non zero constant, the $\left(\begin{array}{c}h_{1}+1 \\ h_{1}-\alpha_{1}+1\end{array}\right) \times\left(\begin{array}{c}h_{2}+1 \\ h_{2}-\alpha_{2}+1\end{array}\right) \times\left(\begin{array}{c}h_{3}+1 \\ h_{3}-\alpha_{3}+1\end{array}\right)$ tensor $\mathcal{T} \in V_{1} \otimes V_{2} \otimes V_{3}$, whose entries are $\mathcal{T}_{I, J, K}$ with lexicographical order of the families $\{I\},\{J\}$, and $\{K\}$ of multi-indices.

\section{The Rank of trifocal Grassmann tensors}

In the classical case of projections from $\mathbb{P}^{3}$ to $\mathbb{P}^{2}$, the rank of the trifocal tensor is known to be 4, (e.g. see [1], [12]), while the rank of the quadrifocal tensor turns out to be 9, [12. Nothing further is known in general about the ranks of Grassmann tensors. In this Section first we provide a canonical form for the matrix (4), in analogy to what was done for the two views case. Then, using this canonical form, we compute $R(\mathcal{T})$ in the general case, i.e. when the center of projections are in general position (see Assumption 5.1). The non general cases are discussed in Section 6 .

5.1. Canonical form. Let $L_{1}, L_{2}$ and $L_{3}$ be the vector spaces of dimension $h_{1}+1, h_{2}+1$ and $h_{3}+1$ respectively, spanned by the columns of $P_{1}^{T}, P_{2}^{T}$ and $P_{3}^{T}$ and let $\Lambda_{1}=\mathbb{P}\left(L_{1}\right), \Lambda_{2}=\mathbb{P}\left(L_{2}\right)$ and $\Lambda_{3}=\mathbb{P}\left(L_{3}\right)$.

We consider, for each triplet of distinct integers $r, s, t=1,2,3$, the following integers:

$$
\begin{aligned}
& i_{r, s}=h_{r}+h_{s}+1-k ; \\
& i=h_{1}+h_{2}+h_{3}+1-2 k ; \\
& j_{r, s}=i_{r, s}-i=k-h_{t} .
\end{aligned}
$$

Our generality assumption is the following:

Assumption 5.1. For any choice of $r, s, t$ with $\{r, s, t\}=\{1,2,3\}$, the intersection $\Lambda_{r s}=L_{r} \cap L_{s}$ with $L_{t}$ span $\mathbb{C}^{k+1}$, or, equivalently, the span of each pair of centers do not intersect the third one.

This assumption implies, in particular, that for any choice of a pair $r, s$, the span of $L_{r}$ and $L_{s}$ is the whole $\mathbb{C}^{k+1}$, or, in other words, that the two centers $C_{r}$ and $C_{s}$ do not intersect. 
Under Assumption 5.1, applying Grassmann formula one sees that the three numbers above have the following meaning: $i_{r, s}=\operatorname{dim}\left(L_{r} \cap\right.$ $\left.L_{s}\right) \geq 0$, for any choice of $r, s, i=\operatorname{dim}\left(L_{1} \cap L_{2} \cap L_{3}\right) \geq 0$ and $j_{r, s}$ is the affine dimension of the center $C_{t}$ i.e. $k-h_{t}=j_{r s}$ for $r, s, t=1,2,3$.

Hence we can choose bases as follows:

$$
\begin{gathered}
L_{1} \cap L_{2} \cap L_{3}=<v_{1}, \ldots, v_{i}> \\
L_{1} \cap L_{2}=<v_{1}, \ldots, v_{i}, w_{1}, \ldots, w_{j_{1,2}}> \\
L_{1} \cap L_{3}=<v_{1}, \ldots, v_{i}, u_{1}, \ldots, u_{j_{1,3}}> \\
L_{2} \cap L_{3}=<v_{1}, \ldots, v_{i}, s_{1}, \ldots, s_{j_{2,3}}>
\end{gathered}
$$

so that:

$$
\begin{aligned}
& L_{1}=<v_{1}, \ldots, v_{i}, w_{1}, \ldots, w_{j_{1,2}}, u_{1}, \ldots, u_{j_{1,3}}> \\
& L_{2}=<v_{1}, \ldots, v_{i}, w_{1}, \ldots, w_{j_{1,2}}, s_{1}, \ldots, s_{j_{2,3}}> \\
& L_{3}=<v_{1}, \ldots, v_{i}, u_{1}, \ldots, u_{j_{1,3}}, s_{1}, \ldots, s_{j_{2,3}}>
\end{aligned}
$$

Through the left action of $G L\left(h_{i}+1\right)$ on $P_{i}, i=1,2,3$, one can assume that the columns of $P_{1}^{T}, P_{2}^{T}$, and $P_{3}^{T}$ are, respectively:

$$
\begin{gathered}
{\left[v_{1}, \ldots, v_{i}, w_{1}, \ldots, w_{j_{1,2}}, u_{1}, \ldots, u_{j_{1,3}}\right],} \\
{\left[v_{1}, \ldots, v_{i}, w_{1}, \ldots, w_{j_{1,2}}, s_{1}, \ldots, s_{j_{2,3}}\right],} \\
{\left[v_{1}, \ldots, v_{i}, u_{1}, \ldots, u_{j_{1,3}}, s_{1}, \ldots, s_{j_{2,3}}\right] .}
\end{gathered}
$$

With this assumption,

$$
\left\{v_{1}, \ldots, v_{i}, w_{1}, \ldots, w_{j_{1,2}}, u_{1}, \ldots, u_{j_{1,3}}, s_{1}, \ldots, s_{j_{2,3}}\right\}
$$

is a basis of $\check{\mathbb{C}}^{k+1}$.

With the right action of $G L(k+1)$ we can reduce (9) to the canonical basis.

With this choice, the matrix (4) becomes the block matrix:

$$
\Phi_{h_{1}, h_{2}, h_{3}}^{k}:=\left[\begin{array}{ccc|ccc|ccc}
I_{i} & \mathbf{0} & \mathbf{0} & I_{i} & \mathbf{0} & \mathbf{0} & I_{i} & \mathbf{0} & \mathbf{0} \\
\mathbf{0} & I_{j_{1,2}} & \mathbf{0} & \mathbf{0} & I_{j_{1,2}} & \mathbf{0} & \mathbf{0} & \mathbf{0} & \mathbf{0} \\
\mathbf{0} & \mathbf{0} & I_{j_{1,3}} & \mathbf{0} & \mathbf{0} & \mathbf{0} & \mathbf{0} & I_{j_{1,3}} & \mathbf{0} \\
\mathbf{0} & \mathbf{0} & \mathbf{0} & \mathbf{0} & \mathbf{0} & I_{j_{2,3}} & \mathbf{0} & \mathbf{0} & I_{j_{2,3}}
\end{array}\right] .
$$


5.2. The rank. The canonical form $\Phi_{h_{1}, h_{2}, h_{3}}^{k}$ of matrix (41) allows one to successfully compute the rank of trifocal Grassmann tensors.

Theorem 5.1. Let $P_{l}: \mathbb{P}^{k} \rightarrow \mathbb{P}^{h_{l}}, l=1,2,3$, be maximal rank projections whose centers satisfy Assumption 5.1. The trifocal Grassmann tensor $\mathcal{T}$ for projections $\left\{P_{l}\right\}$, with profile $\left(\alpha_{1}, \alpha_{2}, \alpha_{3}\right)$, has rank:

$$
\sum_{a_{2}=0}^{j_{12}} \sum_{a_{3}=0}^{j_{13}} \sum_{b_{3}=0}^{j_{23}}\left(\begin{array}{c}
j_{12} \\
a_{2}
\end{array}\right)\left(\begin{array}{c}
j_{13} \\
a_{3}
\end{array}\right)\left(\begin{array}{c}
j_{23} \\
b_{3}
\end{array}\right)\left(\begin{array}{c}
i \\
\alpha_{1}-a_{2}-a_{3}
\end{array}\right)\left(\begin{array}{c}
i-\alpha_{1}+a_{2}+a_{3} \\
\alpha_{2}-j_{12}+a_{2}-b_{3}
\end{array}\right),
$$

where $i=h_{1}+h_{2}+h_{3}+1-2 k$ and $j_{r s}=k-h_{t}$ for $\{r, s, t\}=\{1,2,3\}$.

Proof. Let $\Phi_{h_{1}, h_{2}, h_{3}}^{k}$ be the canonical form of matrix (44) associated to the given projections $P_{l}: \mathbb{P}^{k} \rightarrow \mathbb{P}^{h_{l}}, l=1,2,3$, and let $\left[\Phi_{h_{1}, h_{2}, h_{3}}^{k}\right]_{r}^{s}$ denote the submatrix of $\Phi_{h_{1}, h_{2}, h_{3}}^{k}$ consisting of consecutive columns from column $r$, included, to column $s$, included. To generate each entry of the tensor $\mathcal{T}$ one must choose:

- $a_{1}$ columns from $\left[\Phi_{h_{1}, h_{2}, h_{3}}^{k}\right]_{1}^{i}$,

- $a_{2}$ columns from $\left[\Phi_{h_{1}, h_{2}, h_{3}}^{k}\right]_{i+1}^{i+j_{12}}$,

- $a_{3}$ columns from $\left[\Phi_{h_{1}, h_{2}, h_{3}}^{k}\right]_{i+j_{12}+1}^{i+j_{12}+j_{13}}$,

with $a_{1}+a_{2}+a_{3}=\alpha_{1}$.

Similarly one has to choose:

- $b_{1}$ columns from $\left[\Phi_{h_{1}, h_{2}, h_{3}}^{k}\right]_{i+j_{12}+j_{13}+1}^{2 i+j_{12}+j_{13}}$,

- $b_{2}$ columns from $\left[\Phi_{h_{1}, h_{2}, h_{3}}^{k}\right]_{2 i+j_{12}+j_{13}+1}^{2 i+2 j_{12}+j_{13}}$,

- $b_{3}$ columns from $\left[\Phi_{h_{1}, h_{2}, h_{3}}^{k}\right]_{2 i+2 j_{12}+j_{13}+1}^{2 i+2 j_{12}+j_{13}+j_{23}}$,

with $b_{1}+b_{2}+b_{3}=\alpha_{2}$.

Finally one has to choose:

- $c_{1}$ columns from $\left[\Phi_{h_{1}, h_{2}, h_{3}}^{k}\right]_{2 i+2 j_{12}+j_{13}+j_{23}+1}^{3 i+2 j_{12}+j_{13}+j_{23}}$,

- $c_{2}$ columns from $\left[\Phi_{h_{1}, h_{2}, h_{3}}^{k}\right]_{3 i+2 j_{12}+j_{13}+j_{23}+1}^{3 i+2 j_{12}+2 j_{13}+j_{23}}$,

- $c_{3}$ columns from $\left[\Phi_{h_{1}, h_{2}, h_{3}}^{k}\right]_{3 i+2 j_{12}+2 j_{13}+j_{23}+1}^{3 i+2 j_{12}+2 j_{13}+2 j_{23}}$,

with $c_{1}+c_{2}+c_{3}=\alpha_{c}$.

Moreover to get non vanishing entries of $\mathcal{T}$, the following equalities must be satisfied:

- $a_{1}+b_{1}+c_{1}=i$

- $a_{2}+b_{2}=j_{12}$

- $a_{3}+c_{2}=j_{13}$ 
- $b_{3}+c_{3}=j_{23}$.

From the above conditions, the number of non vanishing entries of the tensor is given by:

$$
\sum_{a_{2}=0}^{j_{12}} \sum_{a_{3}=0}^{j_{13}} \sum_{b_{3}=0}^{j_{23}}\left(\begin{array}{c}
j_{12} \\
a_{2}
\end{array}\right)\left(\begin{array}{c}
j_{13} \\
a_{3}
\end{array}\right)\left(\begin{array}{c}
j_{23} \\
b_{3}
\end{array}\right)\left(\begin{array}{c}
i \\
\alpha_{1}-a_{2}-a_{3}
\end{array}\right)\left(\begin{array}{c}
i-\alpha_{1}+a_{2}+a_{3} \\
\alpha_{2}-j_{12}+a_{2}-b_{3}
\end{array}\right) .
$$

Clearly (12) gives an upper bound for $R(\mathcal{T})$. To prove that (12) is equal to $R(\mathcal{T})$, we use the slices-based characterization of the rank recalled at the end of Section 2.1.

In our case the positions of the non zero entries of $\mathcal{T}$ are different for different faces, i.e. if $T_{\bar{I}, \bar{J}, \bar{K}} \neq 0$, the $T_{\bar{I}, \bar{J}, K}=0$ for all $K \neq \bar{K}$. The reason is that once the columns determined by the multi-indexes $I$ and $J$ are chosen there is at most one possible choice of the columns determined by $K$ which gives a non vanishing minor.

This completes the proof.

The above result is further illustrated by the two following explicit examples.

Example 5.1. In the case of the classical $3 \times 3 \times 3$ trifocal tensor, i.e. of three projections from $\mathbb{P}^{3}$ to $\mathbb{P}^{2}$ with profile $(2,1,1)$, we get: $i=1$ and $i_{r s}=2$ for each $r, s$. Hence, in this case, (10) is:

$$
\Phi_{2,2,2}^{3}:=\left[\begin{array}{ccc|ccc|ccc}
1 & 0 & 0 & 1 & 0 & 0 & 1 & 0 & 0 \\
0 & 1 & 0 & 0 & 1 & 0 & 0 & 0 & 0 \\
0 & 0 & 1 & 0 & 0 & 0 & 0 & 1 & 0 \\
0 & 0 & 0 & 0 & 0 & 1 & 0 & 0 & 1
\end{array}\right] .
$$

The only non vanishing elements of the tensor are: $\mathcal{T}_{131}, \mathcal{T}_{113}, \mathcal{T}_{221}, \mathcal{T}_{312}$, hence $R(\mathcal{T})=4$.

Example 5.2. In the case of three projections from $\mathbb{P}^{4}$ to $\mathbb{P}^{3}, \mathbb{P}^{3}$ and $\mathbb{P}^{2}$, with profile $(2,2,1)$, we get: $i=1, i_{12}=3$, and $i_{13}=i_{23}=2$. Hence, in this case, (10) becomes:

$$
\Phi_{3,3,2}^{4}:=\left[\begin{array}{cccc|cccc|ccc}
1 & 0 & 0 & 0 & 1 & 0 & 0 & 0 & 1 & 0 & 0 \\
0 & 1 & 0 & 0 & 0 & 1 & 0 & 0 & 0 & 0 & 0 \\
0 & 0 & 1 & 0 & 0 & 0 & 1 & 0 & 0 & 0 & 0 \\
0 & 0 & 0 & 1 & 0 & 0 & 0 & 0 & 0 & 1 & 0 \\
0 & 0 & 0 & 0 & 0 & 0 & 0 & 1 & 0 & 0 & 1
\end{array}\right] .
$$

The trifocal tensor $\mathcal{T}$ is a $6 \times 6 \times 3$ tensor and its non vanishing elements are: $\mathcal{T}_{123}, \mathcal{T}_{161}, \mathcal{T}_{213}, \mathcal{T}_{251}, \mathcal{T}_{341}, \mathcal{T}_{431}, \mathcal{T}_{522}, \mathcal{T}_{612}$, hence $R(\mathcal{T})=8$. 
Moreover, one sees that $\mathcal{T}$ is a linear combination of :

$$
\begin{aligned}
& \mathbf{e}_{1}^{1} \otimes \mathbf{e}_{2}^{2} \otimes \mathbf{e}_{3}^{3}, \quad \mathbf{e}_{1}^{1} \otimes \mathbf{e}_{6}^{2} \otimes \mathbf{e}_{1}^{3}, \quad \mathbf{e}_{2}^{1} \otimes \mathbf{e}_{1}^{2} \otimes \mathbf{e}_{3}^{3}, \quad \mathbf{e}_{2}^{1} \otimes \mathbf{e}_{5}^{2} \otimes \mathbf{e}_{1}^{3}, \\
& \mathbf{e}_{3}^{1} \otimes \mathbf{e}_{4}^{2} \otimes \mathbf{e}_{1}^{3}, \quad \mathbf{e}_{4}^{1} \otimes \mathbf{e}_{3}^{2} \otimes \mathbf{e}_{1}^{3}, \quad \mathbf{e}_{5}^{1} \otimes \mathbf{e}_{2}^{2} \otimes \mathbf{e}_{2}^{3}, \quad \mathbf{e}_{6}^{1} \otimes \mathbf{e}_{1}^{2} \otimes \mathbf{e}_{2}^{3},
\end{aligned}
$$

where $\mathbf{e}_{\mathbf{s}}^{\mathbf{r}}$ is the $s$-element of the canonical base of the vector space

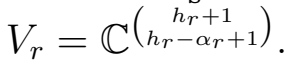

\section{THE NON GENERAL CASE}

In this Section we consider cases in which Assumption 5.1 is not satisfied, and the rank depends on the degenerate geometric configurations of the projections. This is evident also in the simplest case of the classical trifocal tensor for which the rank is 4 for general projections (example 5.1) and becomes 5 when the three centers are on a line (example 6.2).

If Assumption 5.1 is not satisfied one can no longer obtain canonical form (10) for the combined projection matrices, because the integers defined in (6), (77), and (8) lose their geometric meaning and, moreover, (8) may no longer hold.

In this situation one can obtain a different canonical form, from which the rank of the Grassmann tensor can be computed in concrete cases.

We introduce the following notations:

- $g:=\operatorname{dim}\left(L_{1} \cap L_{2} \cap L_{3}\right)$;

- $g_{r s}:=\operatorname{dim}\left(L_{r} \cap L_{s}\right)$;

- $l_{r s}:=g_{r s}-g$;

- $\alpha_{r s}$ the non negative integer such that the span $<L_{r}, L_{s}>$ has dimension $k+1-\alpha_{r s}$;

- $\beta_{r s}$ the non negative integer such that the span $<\Lambda_{r s}, L_{t}>$ has dimension $k+1-\beta_{\text {rs }}$.

By Grassmann formula, these integers are linked to the ones in (66), (7), and (8) as follows: $g=i+\alpha_{r s}+\beta_{r s}$ and $g_{r s}=i_{r s}+\alpha_{r s}$ for any $r, s$.

Arguing as in the previous Section, where $g$ and $l_{r s}$ now play the role of $i$ and $j_{r s}$ respectively, by choosing the first $g+l_{12}+l_{13}+l_{23}$ vectors of the canonical base of $\mathbb{C}^{k+1}$ one gets the following canonical form for the matrix (4), which now depends also on $\alpha_{r s}$ and $\beta_{r s}$.

$$
\Psi_{h_{1}, h_{2}, h_{3}}^{k}:=\left[\begin{array}{cccc|cccc|cccc}
I_{g} & \mathbf{0} & \mathbf{0} & Z_{1}^{1} & I_{g} & \mathbf{0} & \mathbf{0} & Z_{2}^{1} & I_{g} & \mathbf{0} & \mathbf{0} & Z_{3}^{1} \\
\mathbf{0} & I_{l_{1,2}} & \mathbf{0} & Z_{1}^{2} & \mathbf{0} & I_{l_{1,2}} & \mathbf{0} & Z_{2}^{2} & \mathbf{0} & \mathbf{0} & \mathbf{0} & Z_{3}^{2} \\
\mathbf{0} & \mathbf{0} & I_{l_{1,3}} & Z_{1}^{3} & \mathbf{0} & \mathbf{0} & \mathbf{0} & Z_{2}^{3} & \mathbf{0} & I_{l_{1,3}} & \mathbf{0} & Z_{3}^{3} \\
\mathbf{0} & \mathbf{0} & \mathbf{0} & Z_{1}^{4} & \mathbf{0} & \mathbf{0} & I_{l_{2,3}} & Z_{2}^{4} & \mathbf{0} & \mathbf{0} & I_{l_{2,3}} & Z_{3}^{4} \\
\mathbf{0} & \mathbf{0} & \mathbf{0} & Z_{1}^{5} & \mathbf{0} & \mathbf{0} & \mathbf{0} & Z_{2}^{5} & \mathbf{0} & \mathbf{0} & \mathbf{0} & Z_{3}^{5}
\end{array}\right] .
$$


In the matrix $\Psi_{h_{1}, h_{2}, h_{3}}^{k}$, the submatrices $Z_{t}^{p}$, with $t=1,2,3$ and $p=$ $1,2,3,4$ have $\left(h_{t}+1-g-l_{r t}-l_{s t}\right)$ columns. Moreover, by an iterated use of Grassmann formula, one sees that $k+1-g-l_{12}-l_{13}-l_{23}=$ $2\left(\alpha_{r s}+\beta_{r s}\right)-\left(\alpha_{12}+\alpha_{13}+\alpha_{23}\right)$ so that the matrices $Z_{t}^{5}$ have $2\left(\alpha_{r s}+\right.$ $\left.\beta_{r s}\right)-\left(\alpha_{12}+\alpha_{13}+\alpha_{23}\right)$ rows.

Suitable left actions of $G L\left(h_{i}+1\right)$ on the views give the following form for $\Psi_{h_{1}, h_{2}, h_{3}}^{k}$ :

$$
\Psi_{h_{1}, h_{2}, h_{3}}^{k}:=\left[\begin{array}{cccc|cccc|cccc}
I_{g} & \mathbf{0} & \mathbf{0} & \mathbf{0} & I_{g} & \mathbf{0} & \mathbf{0} & \mathbf{0} & I_{g} & \mathbf{0} & \mathbf{0} & \mathbf{0} \\
\mathbf{0} & I_{l_{1,2}} & \mathbf{0} & \mathbf{0} & \mathbf{0} & I_{l_{1,2}} & \mathbf{0} & \mathbf{0} & \mathbf{0} & \mathbf{0} & \mathbf{0} & Z_{3}^{2} \\
\mathbf{0} & \mathbf{0} & I_{l_{1,3}} & \mathbf{0} & \mathbf{0} & \mathbf{0} & \mathbf{0} & Z_{2}^{3} & \mathbf{0} & I_{l_{1,3}} & \mathbf{0} & \mathbf{0} \\
\mathbf{0} & \mathbf{0} & \mathbf{0} & Z_{1}^{4} & \mathbf{0} & \mathbf{0} & I_{l_{2,3}} & \mathbf{0} & \mathbf{0} & \mathbf{0} & I_{l_{2,3}} & \mathbf{0} \\
\mathbf{0} & \mathbf{0} & \mathbf{0} & Z_{1}^{5} & \mathbf{0} & \mathbf{0} & \mathbf{0} & Z_{2}^{5} & \mathbf{0} & \mathbf{0} & \mathbf{0} & Z_{3}^{5}
\end{array}\right] .
$$

The following examples illustrate how, depending on $h_{t}$, the form of $\Psi_{h_{1}, h_{2}, h_{3}}^{k}$ can be further simplified by choosing additional vectors in the canonical basis of $\mathbb{C}^{k+1}$, as columns of the matrices $Z_{t}^{p}$.

Moreover it is clear that the rank of the Grassmann tensor $R(\mathcal{T})$ depends on the entries of the matrices $Z_{t}^{p}$, hence an explicit formula for $R(\mathcal{T})$ is not provided. Nevertheless, as shown in the examples below, in specific concrete cases the number of non vanishing elements of the tensor can be computed, and thus an upper bound for $R(\mathcal{T})$ can be obtained.

Example 6.1. In the case of three projections from $\mathbb{P}^{5}$ to $\mathbb{P}^{2}, \mathbb{P}^{2}$ and $\mathbb{P}^{2}$, with profile $(2,2,2)$, we get: $g=g_{r s}=l_{r s}=0, \alpha_{r s}=0$ and $\beta_{r s}=3$, for each $r, s$. In this case $\Psi_{2,2,2}^{5}$ reduces to $\left[Z_{1}^{5}\left|Z_{2}^{5}\right| Z_{3}^{5}\right]$, where each $Z_{t}^{5}$ is a $(6 \times 3)$ matrix. Up to now we have not yet fixed any vector of the basis, so that, with a further choice of the reference frame, we get:

$$
\Psi_{2,2,2}^{5}:=\left[\begin{array}{ccc|ccc|ccc}
1 & 0 & 0 & 0 & 0 & 0 & z_{11} & z_{12} & z_{13} \\
0 & 1 & 0 & 0 & 0 & 0 & z_{21} & z_{22} & z_{23} \\
0 & 0 & 1 & 0 & 0 & 0 & z_{31} & z_{32} & z_{33} \\
0 & 0 & 0 & 1 & 0 & 0 & z_{41} & z_{42} & z_{43} \\
0 & 0 & 0 & 0 & 1 & 0 & z_{51} & z_{52} & z_{53} \\
0 & 0 & 0 & 0 & 0 & 1 & z_{61} & z_{62} & z_{63}
\end{array}\right]
$$

The trifocal tensor $\mathcal{T}$ is a $3 \times 3 \times 3$ tensor and for generic choices of $z_{i j}$, all its elements are non vanishing and thus no significant upper bound for the rank can be given.

The following example is a degenerate configuration of the classical trifocal tensor.

Example 6.2. In the case of three projections from $\mathbb{P}^{3}$ to $\mathbb{P}^{2}$ with profile $(2,1,1)$ and centers of projection on a line, one has: $g=g_{r s}=2$, 
$l_{r s}=0, \alpha_{r s}=0$ and $\beta_{r s}=1$, for each $r, s$. In this case $\Psi_{2,2,2}^{3}$ reduces to

$$
\Psi_{2,2,2}^{3}:=\left[\begin{array}{ccc|ccc|ccc}
1 & 0 & z_{11} & 1 & 0 & z_{12} & 1 & 0 & z_{13} \\
0 & 1 & z_{21} & 0 & 1 & z_{22} & 0 & 1 & z_{23} \\
0 & 0 & z_{31} & 0 & 0 & z_{32} & 0 & 0 & z_{33} \\
0 & 0 & z_{41} & 0 & 0 & z_{42} & 0 & 0 & z_{43}
\end{array}\right] .
$$

Further changes of coordinates, both in the ambient space and in the views, gives:

$$
\Psi_{2,2,2}^{3}:=\left[\begin{array}{ccc|ccc|ccc}
1 & 0 & 0 & 1 & 0 & 0 & 1 & 0 & 0 \\
0 & 1 & 0 & 0 & 1 & 0 & 0 & 1 & 0 \\
0 & 0 & 1 & 0 & 0 & 0 & 0 & 0 & a \\
0 & 0 & 0 & 0 & 0 & 1 & 0 & 0 & b
\end{array}\right]
$$

with $a$ and $b \neq 0$.

The only non vanishing elements of the tensor are:

$$
T_{113}, T_{131}, T_{212}, T_{221}, T_{311},
$$

hence $R(\mathcal{T})=5$, while the rank of the classical general trifocal is 4 .

6.1. Border ranks. Examples (5.1) and (6.2) seen above, provide evidence, already in the classical setting of projective reconstruction in $\mathbb{P}^{3}$, of the fact that the rank of tensors is not semicontinuous.

Indeed, it is very easy to construct a one dimensional family of triplets of point (centers of projection) which do not lie on a line but converge to a triplet of points on a line. In other words a family of rank 4 tensors which converges to a rank 5 one.

The general situation is still more intricate: even in the first non classical cases of $\mathbb{P}^{4}$ as ambient spaces, we provide some topical examples which display the breadth of phenomena that can occur.

Example 6.3. In the case of three projections from $\mathbb{P}^{4}$ to $\mathbb{P}^{2}, \mathbb{P}^{2}$ and $\mathbb{P}^{2}$, with profile $(2,2,1)$, Assumption 5.1 doesn't hold, and we get: $g=0, g_{r s}=l_{r s}=1, \alpha_{r s}=0$ and $\beta_{r s}=1$, for each $r, s$. In this case $\Psi_{2,2,2}^{4}$ reduces to

$$
\Psi_{2,2,2}^{4}:=\left[\begin{array}{ccc|ccc|ccc}
1 & 0 & 0 & 1 & 0 & 0 & 0 & 0 & z_{13} \\
0 & 1 & 0 & 0 & 0 & z_{22} & 1 & 0 & 0 \\
0 & 0 & z_{31} & 0 & 1 & 0 & 0 & 1 & 0 \\
0 & 0 & z_{41} & 0 & 0 & z_{42} & 0 & 0 & z_{43} \\
0 & 0 & z_{51} & 0 & 0 & z_{52} & 0 & 0 & z_{53}
\end{array}\right] .
$$

Again, a further change of coordinates in the ambient space, gives: 
with $a, b, c \neq 0$.

$$
\Psi_{2,2,2}^{4}:=\left[\begin{array}{ccc|ccc|ccc}
1 & 0 & 0 & 1 & 0 & 0 & 0 & 0 & a \\
0 & 1 & 0 & 0 & 0 & 0 & 1 & 0 & 0 \\
0 & 0 & 0 & 0 & 1 & 0 & 0 & 1 & 0 \\
0 & 0 & 1 & 0 & 0 & 0 & 0 & 0 & b \\
0 & 0 & 0 & 0 & 0 & 1 & 0 & 0 & c
\end{array}\right],
$$

The trifocal tensor $\mathcal{T}$ is a $3 \times 3 \times 3$ tensor and its non vanishing elements are: $\mathcal{T}_{111}, \mathcal{T}_{122}, \mathcal{T}_{131}, \mathcal{T}_{213}, \mathcal{T}_{311}$, from which one easily deduce that $R(\mathcal{T})=4$, because the tensor is a linear combination of:

$$
\left(\mathbf{e}_{1}^{1}+\mathbf{e}_{3}^{1}\right) \otimes \mathbf{e}_{1}^{2} \otimes \mathbf{e}_{1}^{3}, \quad \mathbf{e}_{1}^{1} \otimes \mathbf{e}_{2}^{2} \otimes \mathbf{e}_{2}^{3}, \quad \mathbf{e}_{1}^{1} \otimes \mathbf{e}_{3}^{2} \otimes \mathbf{e}_{1}^{3}, \quad \mathbf{e}_{2}^{1} \otimes \mathbf{e}_{1}^{2} \otimes \mathbf{e}_{3}^{3} .
$$

Starting from the above example, one can consider the following degenerate configurations for lines $C_{A}, C_{B}, C_{C}$, which are centers of projection. Notice that each of these configurations can easily obtained as a limit of a sequence of non degenerate configurations of centers of projection.

a) $C_{A}, C_{B}, C_{C}$ lie in the same hyperplane and no two of them intersect each other;

b) $C_{A}, C_{B}, C_{C}$ span $\mathbb{P}^{4}$ but two of them have nonempty intersection;

c) $C_{A}, C_{B}, C_{C}$ lie in the same hyperplane and two of them have nonempty intersection.

With suitable choices of coordinates and similarly to the rank calculations performed above, one sees that, respectively:

a) $g=g_{r s}, l_{r s}=0$, $\alpha_{r s}=0$ and $\beta_{r s}=2$, for each $r, s$.

In this case $\Psi_{2,2,2}^{4}$ reduces to

$$
\Psi_{2,2,2}^{4}:=\left[\begin{array}{ccc|ccc|ccc}
1 & 0 & 0 & 1 & 0 & 0 & 1 & 0 & 0 \\
0 & 1 & 0 & 0 & 0 & 0 & 0 & z_{11} & z_{12} \\
0 & 0 & 1 & 0 & 0 & 0 & 0 & z_{21} & z_{22} \\
0 & 0 & 0 & 0 & 1 & 0 & 0 & z_{31} & z_{32} \\
0 & 0 & 0 & 0 & 0 & 1 & 0 & z_{41} & z_{42}
\end{array}\right] .
$$

The non vanishing elements of the tensor are:

$$
\mathcal{T}_{113}, \mathcal{T}_{121}, \mathcal{T}_{122}, \mathcal{T}_{131}, \mathcal{T}_{132}, \mathcal{T}_{211}, \mathcal{T}_{212}, \mathcal{T}_{311}, \mathcal{T}_{312}
$$

and $R(\mathcal{T})$ jumps to 5 . With the same notation of example 5.2 , one sees that $\mathcal{T}$ is a combination of:

$$
\begin{aligned}
& \mathbf{e}_{1}^{1} \otimes \mathbf{e}_{1}^{2} \otimes \mathbf{e}_{3}^{3}, \quad \mathbf{e}_{1}^{1} \otimes \mathbf{e}_{2}^{2} \otimes\left(\mathbf{e}_{1}^{3}+\mathbf{e}_{2}^{3}\right), \quad \mathbf{e}_{1}^{1} \otimes \mathbf{e}_{3}^{2} \otimes \mathbf{e}_{1}^{3}, \\
& \mathbf{e}_{2}^{1} \otimes \mathbf{e}_{1}^{2} \otimes\left(\mathbf{e}_{1}^{3}+\mathbf{e}_{2}^{3}\right), \quad \mathbf{e}_{3}^{1} \otimes \mathbf{e}_{1}^{2} \otimes\left(\mathbf{e}_{1}^{3}+\mathbf{e}_{2}^{3}\right) .
\end{aligned}
$$


b) $g=0, g_{12}=l_{12}=2, g_{13}=g_{23}=l_{13}=l_{23}=0$, $\alpha_{12}=2, \beta_{12}=0, \alpha_{13}=\alpha_{23}=0, \beta_{13}=\beta_{23}=2$.

In this case $\Psi_{2,2,2}^{4}$ reduces to

$$
\Psi_{2,2,2}^{4}:=\left[\begin{array}{ccc|ccc|ccc}
1 & 0 & 0 & 1 & 0 & 0 & 0 & z_{11} & z_{12} \\
0 & 1 & 0 & 0 & 1 & 0 & 0 & z_{21} & z_{22} \\
0 & 0 & 1 & 0 & 0 & 0 & 0 & z_{31} & z_{32} \\
0 & 0 & 0 & 0 & 0 & 1 & 0 & z_{41} & z_{42} \\
0 & 0 & 0 & 0 & 0 & 0 & 1 & 0 & 0
\end{array}\right] .
$$

The non vanishing elements of the tensor are:

$$
\mathcal{T}_{123}, \mathcal{T}_{213}
$$

and $R(\mathcal{T})$ drops to 2

c) $g=1, g_{12}=g_{23}=1, l_{12}=l_{23}=0, g_{13}=2, l_{13}=1, \alpha_{12}=\alpha_{23}=$ $0, \beta_{12}=\beta_{23}=2, \alpha_{13}=1, \beta_{13}=1$. In this case $\Psi_{2,2,2}^{4}$ reduces to

$$
\Psi_{2,2,2}^{4}:=\left[\begin{array}{ccc|ccc|ccc}
1 & 0 & 0 & 1 & 0 & 0 & 1 & 0 & 0 \\
0 & 1 & 0 & 0 & 0 & 0 & 0 & 1 & 0 \\
0 & 0 & 1 & 0 & 0 & 0 & 0 & 0 & a \\
0 & 0 & 0 & 0 & 1 & 0 & 0 & 0 & b \\
0 & 0 & 0 & 0 & 0 & 1 & 0 & 0 & c
\end{array}\right] .
$$

The non vanishing elements of the tensor are:

$$
\mathcal{T}_{113}, \mathcal{T}_{121}, \mathcal{T}_{131}, \mathcal{T}_{212}, \mathcal{T}_{311}
$$

$R(\mathcal{T})=4$, and again $\mathcal{T}$ is a linear combination of

$$
\mathbf{e}_{1}^{1} \otimes \mathbf{e}_{1}^{2} \otimes \mathbf{e}_{3}^{3}, \quad \mathbf{e}_{1}^{1} \otimes\left(\mathbf{e}_{2}^{2}+\mathbf{e}_{3}^{2}\right) \otimes \mathbf{e}_{1}^{3}, \quad \mathbf{e}_{2}^{1} \otimes \mathbf{e}_{1}^{2} \otimes \mathbf{e}_{2}^{3}, \quad \mathbf{e}_{3}^{1} \otimes \mathbf{e}_{1}^{2} \otimes \mathbf{e}_{1}^{3} .
$$

In case $a$ ) this shows that the border rank of the tensor is strictly less than its rank, i.e. $\underline{R}(\mathcal{T})<R(\mathcal{T})$.

\section{REFERENCES}

[1] Chris Aholt and Luke Oeding. The ideal of the trifocal variety. Math. Comp., 83(289):2553-2574, 2014.

[2] Alberto Alzati and Alfonso Tortora. Constraints for the trifocal tensor. In Tensors in image processing and computer vision, Adv. Pattern Recognit., pages 261-269. Springer, London, 2009.

[3] Alessandra Bernardi, Enrico Carlini, Maria Virginia Catalisano, Alessandro Gimigliano, and Alessandro Oneto. The hitchhiker guide to: Secant varieties and tensor decomposition. Mathematics, 6(12), 2018.

[4] Marina Bertolini, GianMario Besana, Roberto Notari, and Cristina Turrini. Matrices dropping rank in codimension one and critical loci in computer vision. arXiv:1902.00376, 2019. 
[5] Marina Bertolini, GianMario Besana, and Cristina Turrini. Critical loci for projective reconstruction from multiple views in higher dimension: a comprehensive theoretical approach. Linear Algebra Appl., 469:335-363, 2015.

[6] Marina Bertolini, GianMario Besana, and Cristina Turrini. Generalized fundamental matrices as grassmann tensors. Annali di Matematica Pura ed Applicata (1923 -), 72016.

[7] Marina Bertolini, Roberto Notari, and Cristina Turrini. The Bordiga surface as critical locus for 3-view reconstructions. J. Symbolic Comput., 91:74-97, 2019.

[8] Marina Bertolini and Cristina Turrini. Critical configurations for 1-view in projections from $\mathbb{P}^{k} \rightarrow \mathbb{P}^{2}$. Journal of Mathematical Imaging and Vision, 27:277287, 2007.

[9] Elias Erdtman and Carl Jönsson. Tensor rank. Master's thesis, Linköpings Universitet, Linköpings, Sweden, June 2012.

[10] Richard Hartley and Andrew Zisserman. Multiple view geometry in computer vision. Cambridge University Press, Cambridge, second edition, 2003. With a foreword by Olivier Faugeras.

[11] Richard I. Hartley and Frederik Schaffalitzky. Reconstruction from projections using grassmann tensors. Int. J. Comput. Vision, 83(3):274-293, July 2009.

[12] Anders Heyden. A common framework for multiple view tensors. In Computer VisionECCV'98, pages 3-19. Springer Berlin Heidelberg, 1998.

[13] W. V. D. Hodge and D. Pedoe. Methods of Algebraic Geometry, volume 1. Cambridge University Press, 1994.

[14] J. M. Landsberg. Tensors: geometry and applications, volume 128 of Graduate Studies in Mathematics. American Mathematical Society, Providence, RI, 2012.

[15] Luke Oeding. The quadrifocal variety. Linear Algebra Appl., 512:306-330, 2017.

E-mail address: marina.bertolini@unimi.it

Dipartimento di Matematica "F. Enriques", Università degli Studi di Milano, Via Saldini 50, 20133 Milano, Italy

E-mail address: gbesana@depaul.edu

College of Computing and Digital Media, DePaul University, 243 South Wabash, Chicago IL, 60604 USA

E-mail address: gilberto.bini@unimi.it

Dipartimento di Matematica "F. Enriques", Università degli Studi di Milano, Via Saldini 50, 20133 Milano, Italy

E-mail address: cristina.turrini@unimi.it

Dipartimento di Matematica "F. Enriques", Università degli Studi di Milano, Via Saldini 50, 20133 Milano, Italy 